\title{
ON THE RADIUS OF STARLIKENESS OF CERTAIN ANALYTIC FUNCTIONS
}

\author{
HASSOON S. AL-AMIRI
}

\begin{abstract}
Let $F(z)$ be regular in the unit disk $\Delta:|z|<1$ and normalized by the conditions $F(0)=0$ and $F^{\prime}(0)=1$. Let $f(z)=$ $\frac{1}{2}[z F(z)]^{\prime}$. Recently Libera and Livingston have studied the mapping properties of $f(z)$ when $F(z)$ is known. In particular, they have determined the radius of starlikeness of order $\beta$ for $f(z)$ when $F(z)$ is starlike of order $\alpha, 0 \leqq \alpha \leqq \beta<1$. The author extends this study to include the complementary case $0 \leqq \beta<\alpha$. Also, a different proof has been given to determine the disk in which $\operatorname{Re}\left\{f^{\prime}(z)\right\}>\beta$ when $\operatorname{Re}\left\{F^{\prime}(z)\right\}>\alpha, 0 \leqq \alpha<1,0 \leqq \beta<1$. All results are sharp.
\end{abstract}

1. Introduction. Let $S$ be the class of all regular univalent functions in the unit disk $\Delta:|z|<1$, which are normalized so that $f(0)=0$ and $f^{\prime}(0)=1$. We denote by $K, S^{*}$ and $C$ the subclasses of $S$ which are convex, starlike and close-to-convex, respectively. Libera [4] has shown that if $f(z)$ is in the class $A$, then $F(z)=(2 / z) \int_{0}^{z} f(\xi) d \xi$ is in $A$, where $A$ runs over $K, S^{*}$ and $C$. Livingston [6] has studied the converse problem, namely, the mapping properties of $f(z)$ defined by

$$
f(z)=\frac{1}{2}[z F(z)]^{\prime},
$$

where $F(z)$ is in $K, S^{*}$ or $C$. A typical result is that $f(z)$ is starlike in $|z|<\frac{1}{2}$ when $F(z) \in S^{*}$. Several authors have generalized and refined Livingston's results [1], [2] and [7]. In particular, Padmanabhan [7] refined the results of Livingston by considering the more restricted subclasses $K(\alpha), S^{*}(\alpha)$ and $C(\alpha, \beta)$. The classes $K(\alpha)$ and $S^{*}(\alpha)$, as introduced by Robertson [8], are called the convex functions of order $\alpha$ and the starlike functions of order $\alpha$, respectively. Analytically, $F(z) \in S^{*}(\alpha)$ if and only if $\operatorname{Re}\left\{z F^{\prime}(z) / F(z)\right\}>\alpha, z \in \Delta$. The class $C(\alpha, \beta)$, as introduced by Libera [3], is called the class of close-to-convex functions or order $\alpha$ and type $\beta$. Padmanabhan's main theorem shows that if $F(z) \in S^{*}(\alpha)$, $0<\alpha \leqq \frac{1}{2}$, then $f(z)$, as defined by (1), is starlike of the same order $\alpha$ for

Presented to the Society, January 26, 1973; received by the editors March 5, 1973 and, in revised form, April 20, 1973.

AMS (MOS) subject classifications (1970). Primary 30A32.

Key words and phrases. Univalent functions, starlike functions of order $\alpha$, radius of starlikeness of order $\alpha$, functions with positive real part, extremal function.

(c) American Mathematical Society 1974 
$|z|<\left[\alpha-2+\left(\alpha^{2}+4\right)^{1 / 2}\right] / 2 \alpha$. Analogous results are also obtained when $F(z) \in K(\alpha), 0 \leqq \alpha \leqq \frac{1}{2}$; when $F(z) \in C(\alpha, \beta), 0 \leqq \alpha<1,0 \leqq \beta \leqq \frac{1}{2}$; or in the case where $\operatorname{Re}\left\{F^{\prime}(z)\right\}>\alpha, 0 \leqq \alpha<1$ and $z \in \Delta$.

More recently, Libera and Livingston [5] have obtained the radius of the disk in which $\operatorname{Re}\left\{z f^{\prime}(z) \mid f(z)\right\}>\beta$, for $f(z)$ as given by (1) with $F(z) \in$ $S^{*}(\alpha), 0 \leqq \alpha<1$ and $\beta \geqq \alpha$. Their main theorem (Theorem 1) [5, p. 328] includes Padmanabhan's corresponding result; they extended Padmanabhan's main theorem to include the range $\frac{1}{2}<\alpha<1$. They also obtained the sharp radius of the disk in which $\operatorname{Re}\left\{f^{\prime}(z)\right\}>\beta$, when $\operatorname{Re}\left\{F^{\prime}(z)\right\}>\alpha$, where $f(z)$ and $F(z)$ are related by $(1), \alpha$ and $\beta$ are arbitrary in $[0,1)$ (Theorem 2) [5, p. 333]. Again, for $\alpha=\beta$, Theorem 2 yields the corresponding results of Padmanabhan.

The purpose of this note is to extend Theorem 1 of Libera and Livingston to include the complementary case $0 \leqq \beta<\alpha$. We shall also improve on the content of this theorem by showing that Theorem 1 holds for $\beta_{0}(\alpha) \leqq$ $\beta<1$ and $\beta_{0}(\alpha)<\alpha$. Central to our approach is a theorem due to V. A. Zmorovič [10]. This theorem turned out to be just as effective in providing a new proof for Theorem 2 .

2. Preliminaries. We shall denote by $P$ the class of regular functions $p(z)$ in $\Delta$ with $p(0)=1$ such that $\operatorname{Re}\{p(z)\}>0$. If $F(z) \in S^{*}(\alpha)$, then there exists $p(z) \in P$ such that

$$
z F^{\prime}(z) / F(z)=\alpha+(1-\alpha) p(z) .
$$

From (1) we get

$$
\left(z f(z)-\int_{0}^{z} f(\xi) d \xi\right) / \int_{0}^{z} f(\xi) d \xi=z F^{\prime}(z) / F(z) .
$$

Substitute (2) in the above to get $z f(z) / \int_{0}^{z} f(\xi) d \xi=1+\alpha+(1-\alpha) p(z)$. Thus

$$
\begin{aligned}
& z f^{\prime}(z) / f(z)-\beta \\
& \quad=-(1+\beta)+(1-x)(h+p(z))+z p^{\prime}(z) /(h+p(z))
\end{aligned}
$$

where $h=(1+\alpha) /(1-\alpha), 0 \leqq \alpha<1$.

In $\$ 3$ we shall determine $r_{\alpha, \beta}$ the radius of starlikeness of order $\beta$ for $f(z)$, i.e., the radius of the largest disk in which $\operatorname{Re}\left\{z f^{\prime}(z) / f(z)-\beta\right\}>0$. Clearly, (3) implies that $r_{\alpha, \beta}$ is the smallest positive root of $Q_{\alpha, \beta}(r)=0$, where

(4) $Q_{\alpha, \beta}(r)=\min _{p \in P^{\prime}|z|=r<1} \operatorname{me}\left\{-(1+\beta)+(1-\alpha)(h+p(z))+\frac{\left(z p^{\prime}(z)\right.}{h+p(z)}\right\}$. 
This extremal problem is a special case of

$$
Q(r)=\min _{p \in P|z|=r<1} \min \left\{\Psi\left(p(z), z p^{\prime}(z)\right)\right\},
$$

where $\Psi(w ; W)$ is an analytic function of the variables $w$ and $W$ in the $W$-plane and in the half plane $\operatorname{Re}\{w\}>0$. It is known [9] that the minimum in $(5)$ is realized for functions of the form

$$
p(z)=\lambda_{1} \frac{1+z e^{-i \theta_{1}}}{1-z e^{-i \theta_{1}}}+\lambda_{2} \frac{1+z e^{-i \theta_{2}}}{1-z e^{-i \theta_{2}}},
$$

where $\theta_{1}, \theta_{2} \in[0,2 \pi], \lambda_{1}, \lambda_{2} \geqq 0$ and $\lambda_{1}+\lambda_{2} \geqq 0$ and $\lambda_{1}+\lambda_{2}=1$. In $\S 3$ we obtain $r_{\alpha . \beta}$ through an application of a theorem due to V. A. Zmorovič [10] which is stated next.

TheOREM A (V. A. ZMOROVIČ). Let $\Psi(w ; W)=M(w)+N(w) W$, where $M(w)$ and $N(w)$ are defined and finite in the half plane $\operatorname{Re}\{w\}>0$. Put

$$
w=\lambda_{1} \frac{1+z_{1}^{m}}{1-z_{1}^{m}}+\lambda_{2} \frac{1+z_{2}^{m}}{1-z_{2}^{m}}, \quad W=\lambda_{1} \frac{2 m z_{1}^{m}}{\left(1-z_{1}^{m}\right)^{2}}+\lambda_{2} \frac{2 m z_{2}^{m}}{\left(1-z_{2}^{m}\right)^{2}},
$$

where $z_{1}$ and $z_{2}$ are arbitrary points on $|z|=r<1, m$ is a positive integer, $\lambda_{1} \geqq 0, \lambda_{2} \geqq 0, \lambda_{1}+\lambda_{2}=1$. Then the function $\Psi(w, W)$ has the form

$$
\Psi(w ; W)=M(w)+\frac{1}{2} m\left(w^{2}-1\right) N(w)+\frac{1}{2} m\left(\rho^{2}-\rho_{0}^{2}\right) N(w) e^{2 i w},
$$

where

$$
\begin{array}{rr}
\left(1+z_{k}^{m}\right) /\left(1-z_{k}^{m}\right)=a+\rho \exp \left(i \psi_{k}\right) & (k=1,2), \\
w=a+\rho_{0} \exp \left(i \psi_{0}\right) & \left(0 \leqq \rho_{0} \leqq \rho\right), \\
\left|z_{1}\right|=\left|z_{2}\right|=r, \quad a=\left(1+r^{2 m}\right) /\left(1-r^{2 m}\right), \\
\rho=2 r^{m} /\left(1-r^{2 m}\right), \quad e^{i \psi}=i \exp \left[i\left(\psi_{1}+\psi_{2}\right) / 2\right] .
\end{array}
$$

Also for fixed $w(|w-a|<\rho)$ the angle $2 \psi$ in the above formula can take all the values from $[0,2 \pi]$; thus

$$
\begin{aligned}
\min \operatorname{Re}\{\Psi(w ; W)\} & =\Psi_{p}(w) \\
= & \operatorname{Re}\left\{M(w)+\frac{1}{2} m\left(w^{2}-1\right) N(w)\right\}-\frac{1}{2} m|N(w)|\left(\rho^{2}-\rho_{0}^{2}\right) .
\end{aligned}
$$

The minimum in (7) is reached when

$$
\exp [i(2 \psi+\arg N(w))]=-1 .
$$

Comparing problem (4) with the terms of Theorem A, we have $m=1$, 
and

$$
\begin{aligned}
M(w) & =-(1+\beta)+(1-\alpha)(w+h), \\
N(w) & =1 /(w+h), \\
h & =(1+\alpha) /(1-\alpha), \quad 0 \leqq \alpha<1 .
\end{aligned}
$$

From (4), (7) and these relations our problem is reduced to minimizing $\Psi_{\rho}\left(w^{\prime}\right)$, where

(9) $\Psi_{\rho}(w)=\operatorname{Re}\left\{-(1+\beta)+(1-\alpha)(w+h)+\frac{1}{2} \frac{w^{2}-1}{w+h}\right\}-\frac{1}{2|w+h|}$.

\section{Two theorems.}

THEOREM 1. Let $F(z)$ be a function in $S^{*}(\alpha), 0 \leqq \alpha<1, f(z)=\frac{1}{2}[z F(z)]^{\prime}$ for $z \in \Delta$. Let $r_{\alpha, \beta}$ be the radius of the largest disk in which $\operatorname{Re}\left\{z f^{\prime}(z) \mid f(z)\right\}>$ B. Put

$$
\begin{aligned}
& a^{2}+2(\beta-2 \alpha-1) a+(2 h+\beta+1)^{2}-4(2-\alpha) h^{2}=0 \\
& \alpha(2 \alpha-\beta-1) r^{2}+(2(2 \alpha-1)-\beta(1+\alpha)) r+1-\beta=0
\end{aligned}
$$

where $a=\left(1+r^{2}\right) /\left(1-r^{2}\right), h=(1+\alpha) /(1-\alpha)$; also

$$
A_{1} \beta^{3}-A_{2} \beta^{2}+A_{3} \beta-A_{4}=0
$$

where

$$
\begin{array}{ll}
A_{1}=4(2-\alpha)\left(1-\alpha^{2}\right), & A_{2}=(1+\alpha)\left(4 \alpha^{3}-12 \alpha^{2}+23 \alpha-33\right), \\
A_{3}=2\left(6 \alpha^{3}-8 \alpha^{2}-52 \alpha+18\right), & A_{4}=4\left(8 \alpha^{4}-12 \alpha^{3}-16 \alpha^{2}+12 \alpha-1\right) .
\end{array}
$$

Then $r_{\alpha, \beta}$ is the smallest positive root $r$ in $(10)$ with $0 \leqq \beta \leqq \beta_{0}(\alpha)$ and the smallest positive root $r$ in $(11)$ with $\beta_{0}(\alpha) \leqq \beta<1$, where $\beta_{0}(\alpha)$ is the smallest positive root of (12). These results are sharp.

Proof. As remarked in $\$ 2$, our problem is reduced to the extremal problem given by (9). Equality holds in (9) when the point $w(|w-a|<\rho)$ is fixed, and the chord passing through it and through the points $a+\rho \exp i \psi_{k}(k=1,2)$ is perpendicular to the vector $\exp i \phi / 2$, where $w+h=R \exp i \phi$ (this follows from (8)). Let $\|=a+\xi+i \eta, \rho_{0}^{2}=\xi^{2}+\eta^{2} \leqq \rho^{2}$. Then (9) becomes

$$
\begin{aligned}
\Psi_{\rho}(w) \equiv & \Psi_{\rho}(\xi ; \eta)=-(1+\beta+h)+(3-\alpha)(a+\xi+h) \\
& +\frac{1}{2}\left(h^{2}-1\right)(a+\xi+h) R^{-2}-\frac{1}{2}\left(\rho^{2}-\xi^{2}-\eta^{2}\right) R^{-1},
\end{aligned}
$$


where $R^{2}=(a+\xi+h)^{2}+\eta^{2}$. One can easily show that $\partial \Psi_{\rho} / \partial \eta=$ $\frac{1}{2} \eta R^{-4} S(\xi ; \eta)$, where

$$
\begin{aligned}
S(\xi ; \eta)= & \left(\xi^{2}+4(a+h) \xi^{2}+\rho^{2}+\eta^{2}+2\left(a+h^{2}\right)\right) R \\
& -2\left(h^{2}-1\right)(a+\xi+h) \\
\geqq & {\left[\xi^{2}+4(a+h) \xi+\rho^{2}+2(a+h)^{2}-2\left(h^{2}-1\right)\right](\xi+a+h) . }
\end{aligned}
$$

The minimum on the interval $-\rho \leqq \xi \leqq \rho$ of the expression in the last square brackets is reached at $\xi=-\rho$ and equals $2(a-\rho)^{2}+4 h(a+\rho)+$ $2>0$. This shows that the minimum of $\Psi_{\rho}(\xi ; \eta)$ on every chord $\xi=$ constant is reached when $\eta=0$. Therefore, the minimum of $\Psi_{\rho}(\xi ; \eta)$ in the circle $\xi^{2}+\eta^{2} \leqq \rho^{2}$ is reached on the diameter $\eta=0$. Set $\eta=0$ and $R=a+\xi+h$ in (13), we arrive at the following:

(14) $\psi_{\rho}(\xi ; 0) \equiv l(R)=(2-\alpha) R+\left(h^{2}+a h\right) R^{-1}-a-2 h-\beta-1$.

Simple calculations show that the absolute minimum of $l(R)$ is realized at

$$
R_{0}=\left[\left(h^{2}+a h\right) /(2-\alpha)\right]^{1 / 2} .
$$

Since $R_{0}^{2}=\left[\left(h^{2}+a h\right) /(2-\alpha)\right]<h^{2}+a h<(a+h+\rho)^{2}, \quad R_{0}<a+h+\rho$. However, if $R_{0} \notin[a+h-\rho, a+h+\rho]$, then the minimum of $l(R)$ is attained at

$$
R_{1}=a+h-\rho .
$$

The radius $r_{\alpha, \beta}$ is therefore determined from either

$$
l\left(R_{0}\right)=0 \text {, }
$$

where $R_{0}$ is given by (15), or from

$$
l\left(R_{1}\right)=0
$$

where $R_{1}$ is given by (16).

These two equations coincide for some $\beta_{0}=\beta_{0}(\alpha)\left(\beta_{0}(\alpha)\right.$ will be determined later). One may verify that (17) and (18) can be reduced to (10) and (11), respectively. However, from (10) and (11) we get,

$$
r_{1}=r_{\alpha, \beta}=\left(\left(2 \alpha-\beta-\delta^{1 / 2}\right) /\left(2 \alpha-\beta+2-\delta^{1 / 2}\right)\right)^{1 / 2},
$$

where $\delta=(\beta-2 \alpha-1)^{2}+4(2-\alpha) h^{2}-(2 h+\beta+1)^{2}$, and

$$
\begin{aligned}
r_{2}=r_{\alpha, \beta}=2(1-\beta) /[ & (\beta(1+\alpha)-2(2 \alpha-1)) \\
+ & \left((\beta(1+\alpha)-2(2 \alpha-1))^{2}\right. \\
& \left.-4 \alpha(1-\beta)(2 \alpha-\beta-1))^{1 / 2}\right],
\end{aligned}
$$

respectively. To determine the $\beta_{0}=\beta_{0}(\alpha)$ that makes the transition from (19) to (20) set $R_{0}=R_{1}$. Thus $R_{0}=\left(\left(h^{2}+a h\right) /(2-\alpha)\right)^{1 / 2}=a-\rho+h=R_{1}$. 
It follows then

$$
h^{2}+a h=(2-\alpha) R_{1}^{2} .
$$

From (18), (14) and (21), we obtain $2(2-\alpha) R_{1}=a+2 h+\beta+1$. Thus

$$
(3-2 \alpha) a+2 \alpha-\beta+1=2(2-\alpha) \rho \text {. }
$$

Squaring both sides of (22), replacing $\rho^{2}$ by $a^{2}-1$ then using (10) to eliminate $a^{2}$, we finally arrive at

$$
a=\frac{(1-\alpha) \beta^{2}+(\alpha+5) \beta-\left(2 \alpha^{2}+7 \alpha-3\right)}{(1-\alpha)(2 \alpha-\beta+1)} .
$$

Equation (12) is now easily deduced from (10) and (23).

Note that $r_{\alpha, \beta}=r_{1}$ as given by (19) cannot be used when $\beta>\alpha$, since then $\delta<0$ and $r_{1}$ becomes a nonreal number. Therefore, $r_{\alpha, \beta}=r_{2}$ may be used for $\beta>\alpha$. In fact $r_{2}$ may be used for $\beta \geqq \alpha$ (see [5]). Since $\beta=\alpha$ is not a root for (12) then $\beta_{0}(\alpha)<\alpha$.

Now we determine the extremal functions $f_{0}(z)$ for Theorem 1. Taking into account (8) and the fact that the minimum in case (17) is reached at a point on the diameter $\eta=0$ (not an end point), we can conclude that $p(z)$ (see (6)) should in this case be taken in the form

where $\theta$ is given by

$$
p(z)=\frac{1}{2} \cdot \frac{1+z e^{-i \theta}}{1-z e^{-i \theta}}+\frac{1}{2} \cdot \frac{1+z e^{i \theta}}{1-z e^{i \theta}},
$$

$$
R_{0}=\operatorname{Re}\{h+w\}=h+\left(1-r_{1}^{2}\right)\left(1-2 r_{1} \cos \theta+r_{1}^{2}\right)^{-1},
$$

where $r_{1}$ is given by (19) and $R_{0}$ is given by (15). From (2), (1) and the above the extremal function is

$$
f_{0}(z)=z\left(1-2 \alpha z \cos \theta+\alpha z^{2}\right)\left(1-2 z \cos \theta+z^{2}\right)^{x-2} .
$$

In case (18), the minimum is realized at an end point of the diameter $\eta=0$. Thus $p(z)$ is given by $p(z)=(1+z) /(1-z)$. Again the extremal function is

$$
f_{0}(z)=z(1-\alpha z)(1-z)^{2 \alpha-3} \text {. }
$$

The proof of Theorem 1 is now completed.

In the remaining part of this section we give a different proof for Theorem 2 of Libera and Livingston [5, p. 333]. The method is the same one employed in proving Theorem 1 above. We adopt the notations of Libera and Livingston and hence we restate their theorem.

THEOREM 2. For $0 \leqq \alpha, \beta<1$ and $0<r<1$ let

$$
N_{1}(r)=(1-\beta)+(3 \alpha-2 \beta-1) r+(2 \alpha-\beta-1) r^{2}
$$


and

$$
M_{1}(r)=(9 \alpha-8 \beta-1)+(6-22 \alpha+16 \beta) r^{2}+(17 \alpha-8 \beta-9) r^{4} .
$$

If $F(z)$ and $f(z)$ are related as in (1) and $\operatorname{Re}\left\{F^{\prime}(z)\right\}>\alpha, z \in \Delta$, then $\operatorname{Re}\left\{f^{\prime}(z)\right\}>\beta$ for $|z|<r_{0}$, where $r_{0}$ is the smallest positive root of the equation

$$
\begin{aligned}
N_{1}(r)=0, & \text { when } \beta \geqq \alpha, \text { or } \\
& \beta<\alpha \text { and } \alpha \leqq(8-3 \sqrt{ } 7) /(16-5 \sqrt{ } 7), \text { or } \\
& ((16-5 \sqrt{ } 7) \alpha+3(\sqrt{ } 7-8)) /(8-2 \sqrt{ } 7) \leqq \beta<\alpha \\
& \text { and }(8-3 \sqrt{ } 7) /(16-5 \sqrt{ } 7)<\alpha ;
\end{aligned}
$$

and $r_{0}$ is the root greater than and closest to $(\sqrt{ } 7-2)$ of the equation $M_{1}(r)=0$, when $\beta<((16-5 \sqrt{ } 7) \alpha+(3 \sqrt{ } 7-8)) /(8-2 \sqrt{ } 7)$ and

These results are sharp.

$$
\alpha>(8-3 \sqrt{ } 7) /(16-5 \sqrt{ } 7) \text {. }
$$

Proof. If $\operatorname{Re}\left\{F^{\prime}(z)\right\}>\alpha$, then there is $p(z) \in P$ such that

$$
F^{\prime}(z)=\alpha+(1-\alpha) p(z) .
$$

Equations (1) and (29) yield $2\left(f^{\prime}(z)-\beta\right)=2(\alpha-\beta)+(1-\alpha)\left(2 p(z)+z p^{\prime}(z)\right)$. Now $r_{0}$ becomes the smallest positive root of $Q_{\alpha, \beta}(r)=0$, where

$$
Q_{\alpha, \beta}(r)=\min _{p \in P \mid} \min _{|z|=r<1} \operatorname{Re}\left\{2(\alpha-\beta)+2(1-\alpha) p(z)+(1-\alpha) z p^{\prime}(z)\right\}
$$

On comparing (30) with the terms of Theorem A, we then have $m=1$, $M(w)=2(\alpha-\beta)+2(1-\alpha) w, N(w)=1-\alpha$. From $(30),(7)$ and the above relations, our problem is reduced to finding the solution of the extremal problem:

$$
\begin{aligned}
\min \operatorname{Re}\{\Psi(w ; W)\}= & \operatorname{Re}\left\{2(\alpha-\beta)+2(1-\alpha) w+\frac{1}{2}(1-\alpha)\left(w^{2}-1\right)\right\} \\
& -\frac{1}{2}(1-\alpha)\left(\rho^{2}-\rho_{0}^{2}\right) .
\end{aligned}
$$

As before, let $w=a+\xi+i \eta, \operatorname{Re}\left\{w^{2}\right\}=(a+\xi)^{2}-\eta^{2}$, then (31) yields

$$
\operatorname{Re}\{\Psi(w ; W)\} \equiv \Psi_{\rho}(\xi)=2(\alpha-\beta)+(1-\alpha)\left(2 a+2 \xi+a \xi+\xi^{2}\right) .
$$

From $d \Psi_{\rho} / d \xi=(2+2 \xi+a)(1-\alpha)$ it follows that the absolute minimum of $\Psi_{\rho}(\xi)$ is achieved at

$$
\xi_{0}=-(a+2) / 2
$$

We note that $\xi_{0}<\rho$, however $\xi_{0}>-\rho$ only if

$$
\frac{1}{2}\left(\left(1+r^{2}\right) /\left(1-r^{2}\right)+2\right)<2 r /\left(1-r^{2}\right) .
$$


Hence $\xi_{0}>-\rho$ when $r^{2}+4 r-3>0$, that is if $r>\sqrt{ } 7-2$. In this case

$$
\min _{|z|=r} \Psi_{\rho}(\xi)=\Psi_{\rho}\left(\xi_{0}\right)=(1-\alpha) a^{2}+4(1-\alpha) a+(r-12 \alpha+8 \beta),
$$

with $r>\sqrt{ } 7-2$. Otherwise, the minimum of (32) is achieved at $\xi_{1}=-\rho$. For this case we obtain

$$
\Psi_{\rho}\left(\xi_{1}\right)=2(\alpha-\beta)+2(1-\alpha)(a-\rho)+(1-\alpha)\left(\rho^{2}-a \rho\right),
$$

provided $r \leqq \sqrt{ } 7-2$. Therefore $r_{0}$ is the smallest positive root of $\Psi_{\rho}\left(\xi_{0}\right)=0$ for $r>\sqrt{ } 7-2$, or $\Psi_{\rho}\left(\xi_{1}\right)=0$ for $r<\sqrt{ } 7-2$. Note that upon replacing $a$ and $\rho$ in terms of $r$ in (34) and (35), $r_{0}$ becomes the smallest positive root of

$$
\begin{array}{ll}
M_{1}(r)=0 & \text { when } r>\sqrt{ } 7-2, \text { or } \\
N_{1}(r)=0 & \text { when } r \leqq \sqrt{ } 7-2,
\end{array}
$$

where $M_{1}(r)$ and $N_{1}(r)$ as given by (27) and (26), respectively. Thus the main part of the theorem is completed. The analysis of the several possible cases which are stated in the theorem can be carried out in exactly the same manner as in Libera and Livingston [5].

Now to determine the extremal functions, it is sufficient to determine $p(z)$, as given by (6), since then the extremal functions follow from (29) and (1). As before when $r>\sqrt{ } 7-2$

$$
p(z)=\frac{1}{2} \frac{1+z e^{-i \theta}}{1-z e^{-i \theta}}+\frac{1}{2} \frac{1+z e^{i \theta}}{1-z e^{i \theta}},
$$

where $\theta$ is given by

$$
\begin{aligned}
\xi_{0} & =-(a+2) / 2=\operatorname{Re}\{w-a\} \\
& =-a+\left(1-r^{2}\right) /\left(1-2 r \cos \theta+r^{2}\right),
\end{aligned}
$$

and $r$ is the smallest positive root for $M_{1}(r)=0$. Further simplifications show that

$$
\theta=\arccos \left(3-6 r^{2}-r^{4}\right) / 2 r\left(1-3 r^{2}\right) .
$$

As for the case $r<\sqrt{ } 7-2, p(z)=(1+z) /(1-z)$. The proof of Theorem 2 is now completed.

\section{REFERENCES}

1. H. S. Al-Amiri, On the radius of univalence of certain classes of analytic functions, Colloq. Math. 28 (1973).

2. S. D. Bernardi, The radius of univalence of certain analytic functions, Proc. Amer. Math. Soc. 24 (1970), 312-318. MR 40 \#433.

3. R. J. Libera, Some radius of convexity problems, Duke Math. J. 31 (1964), 143-158. MR 28 \#4099.

4. - Some classes of regular univalent functions, Proc. Amer. Math. Soc. 16 (1965), 755-758. MR 31 \#2389. 
5. R. J. Libera and A. E. Livingston, On the univalence of some classes of regular functions, Proc. Amer. Math. Soc. 30 (1971), 327-336. MR 44 \#5442.

6. A. E. Livingston, On the radius of univalence of certain analytic functions, Proc. Amer. Math. Soc. 17 (1966), 352-357. MR 32 \#5861.

7. K. S. Padmanabhan, On the radius of univalence of certain classes of analytic functions, J. London Math. Soc. (2) 1 (1969), 225-231. MR 40 \#331.

8. M. S. Robertson, On the theory of univalent functions, Ann. of Math. (2) 37 (1936), 374-408.

9. - Variational methods for functions with positive real part, Trans. Amer. Math. Soc. 102 (1962), 82-93. MR 24 \#A3288.

10. V. A. Zmorovič, On bounds of convexity for starlike functions of order $\alpha$ in the circle $|z|<1$ and in the circular region $0<|z|<1$, Mat. Sb. 68 (110) (1965), 518-526; English transl., Amer. Math. Soc. Transl. (2) 80 (1969), 203-213. MR 33 \#5875.

Department of Mathematics, Bowling Green State University, Bowling Green, OHIO 43403 